\title{
Thermal analysis of polymers
}

\author{
Thomas R.MANLEY \\ Department of Mechanical Engineering and Manufacturing Systems, Newcastle Polytechnic, \\ Ellison Building, Newcastle upon Tyne, NE1 8ST, U.K.
}

\begin{abstract}
Before 1963 there was little thermal analysis of polymers in the U.K. Since then DTA, DSC and TG have become widely used. The combination of TA techniques with other techniques including Mass Spectroscopy and Fourier Transform Infra Red spectroscopy (FTIR) provide especially powerful analytical tools.

The sensitivity of TA techniques has increased. Computers have had a marked impact on the operation of TA instruments and also on the manipulation of results.

Thermomechanical techniques are being increasingly used as plastics and rubbers find applications in engineering.

The thermal degradation of polymers remains a major concern and an alternative method of studying the decomposition of polymers is described.
\end{abstract}

\section{INTRODUCTION}

Heat was used in the earliest days of science; thermal analysis may be said to have been in use in biblical times (St. Paul: "Fire shall try every man's work" COR. 1, 111, 13) and even earlier, Iron Age Britons have left traces of knowledge of thermal properties. Attempts have been made to date thermal analysis from the 18th century through the work of Joseph Black and Josiah Wedgewood but true DTA had to wait for Roberts-Austen and TG for Honda at the beginning of the 20th century.

Historical evidence for plastic materials is even older. The mother of Moses used bitumen and bullrushes to make a craft for her baby. This principle of a composite of a resin and fibrous reinforcement is used today in the Royal Navy minesweepers that patrol the Persian Gulf. The beginning of the plastics industry can be dated to 1851; at the Crystal Palace Exhibition in London on that date "Parkesine" a cellulose nitrate plastic was shown in several moulded articles. The natural polymer "lac" has been used in India for thousands of years as a varnish (lacquer) and was moulded at the end of the 19th century. Rubber was used in Central America before the time of Columbus and in 1820 Thomas Hancock discovered that mastication (shearing) of coagulated rubber made it plastic, whilst heating the plastic rubber with sulphur regenerated elastic material.

As with Thermal analysis however it is only in the 20th century that we can talk about the Polymer Age. The application of TA to polymers had to await the second half of the 20th century. After the Second World War there was some work in the US and the first paper in the UK was given at a Society of Chemical Industry Conference in 1962 [1].

In the quarter century since that paper was published the TA of polymers has changed out of all recognition. In 1962 DTA was conducted on apparatus made in individual laboratories; nowadays the vast majority of workers use commercial equipment. TGA and DTA were the only techniques used. Home-made equipment does however still have its uses where explosive or corrosive materials are being investigated as the cheap lab made equipment is often adequate for these reactions as a good deal of energy is available. In the intervening years DSC has come to the fore, both in the original form of power compensated DSC or differential enthalpy analysis, and as heat flux DSC. In the polymer field where little work is done above $400^{\circ} \mathrm{C}$, DTA has been substantially replaced by DSC. The use of high pressure DSC has enabled significant advances to be made in studies on crystallisation and curing [2]. 
Improvements in instrumentation over the period in question are striking; amplifiers consisting of bulky boxes and photo galvanometers are now more likely to be found in a museum than in a TA laboratory. The use of TA to study kinetics has been a fruitful field which was opened up with the improvements in instrumentation. Initially DTA could only be considered as a qualitative technique but this has now completely changed and TA methods are becoming widely accepted as the basis for British, European and American Standards. DTA alone was long regarded as an indicative technique and instruments to combine DTA and TG soon appeared. Other combinations have been investigated and the difficult problems of "interfacing" have been overcome.

Interfacing of DTA with a mass spectrometer was one of the first combined methods. A great deal of work has been done on this by Stanton Redcroft and some unique information has been obtained on fibres and polymers both as resins and finished products.

Evolved gas analysis has provided a great deal of information especially on the decomposition of polymers; evolved gas analysis as low pressures, the so-called thermal vaporisation analysis has been extensively used by N. Grassie and I.C. McNeill at Glasgow.

When evolved gas analysis is interfaced with Fourier Transform infra red spectroscopy (FTIR) a new dimension is added to TA which could be one of the most fruitful combinations ever employed. EGA and TG and EGA/DTA have been employed to provide further means of studying decomposition reactions.

Thermo mechanical measurements have come from being of negligible use to one of the most widely used techniques in the elucidation of the structures and properties of polymers. Thermo mechanical methods may be divided into thermodilatometry, static methods (TMA) and dynamic methods (DTMA: dynamic mechanical thermal analysis) and torsional techniques (TBA: torsional braid analysis) and the torsional pendulum.

The torsional pendulum is probably the oldest form of DMA and the modified form of torsional braid analyser TBA has provided new insights into the methods of measuring the physical properties of polymers and the fundamental behaviour during the process of curing.

The most significant change in TA techniques however has resulted from advances in computing. Computers are now used to operate TA equipment, to improve the sensitivity of instruments by manipulation of results and to interpret the resultant traces.

Other changes that have occurred include the use of energy sources other than heat e.g. in photopolymerisation; [3] the study of explosions and highly exothermic reactions in special equipment.

In the future lies the use of FTIR directly measuring the changes taking place in a solid specimen and incidentally providing a true measure of temperature.

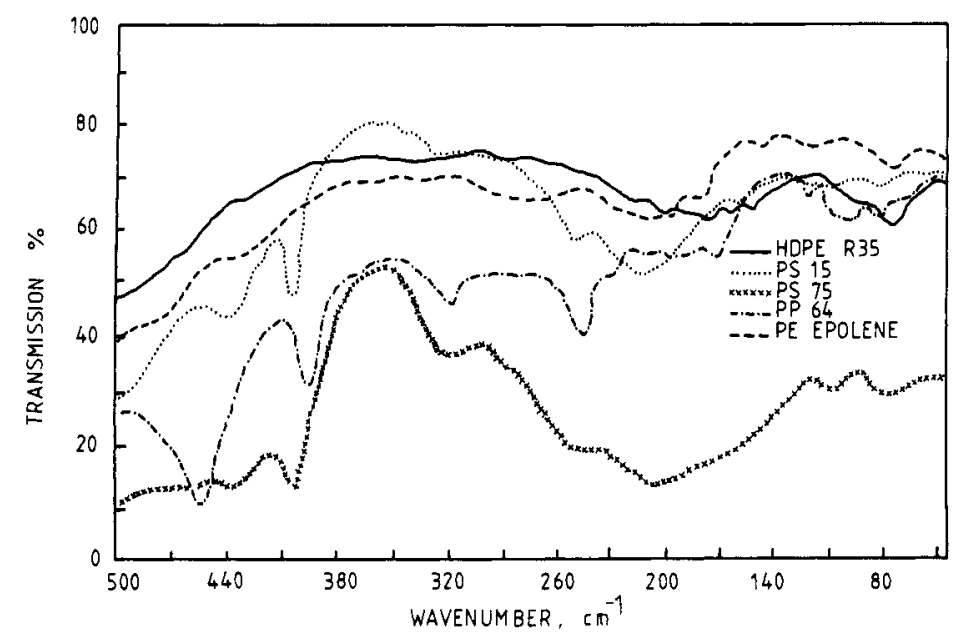

FIG 1.

FIRST FTIR SPECTRA OF POLYMERS 


\section{COMBINED TECHNIQUES}

Considerable synergy is obtained by the combination of DTA, DSC or TG with other techniques.

This is exemplified by considering the combinations of TG and FTIR; TG and Mass Spectroscopy; and DTA and smoke estimation measurements [4].

\section{TG/FTIR}

FTIR utilizes an interferometer constructed on principles initiated by Michelson a century ago. He himself could only make visual observations and the value of FTIR only became apparent with improvements in metrology and computing.

The major advantage of an interferometer is the greatly improved signal to noise ratio [5]. This improvement can be used to give higher resolution, or to obtain spectra where little energy is available, as in astronomy or in studying the far infra red region or to obtain spectra rapidly.

For example it was only by the use of FTIR that the spectra of polymers in the far infra red could be obtained. The first such spectra were obtained in 1965 and are shown in Fig 1. [6].

The standard for TG is calcium oxalate and Compton has shown the infra red peaks obtained corresponding to the weight losses in the TG trace. These are readily identified as being the expected emissions of water, carbon monoxide and carbon dioxide. In addition however FTIR shows that some carbon dioxide is produced before the carbon monoxide is detected. This suggests that the decomposition of calcium oxalate is not so clear cut as had been thought and also it illustrates the sensitivity of FTIR.

In Fig 2 DW/DT is the first derivative of weight loss against temperature (or DTG). This correlates with the evolved gas profile (EGP) but the peak sizes are different because the infra red absorption of the evolved gas is the major factor controlling the EGP [7].

The spectral regions (windows) at $1780-1900,2050-2200$ and $2300-2380 \mathrm{~cm}^{-1}$ show the evolution during the experiment of water, carbon monoxide and carbon dioxide respectively. The emission of some $\mathrm{CO}_{2}$ at the time of the $\mathrm{CO}$ evolution is clearly demonstrated.
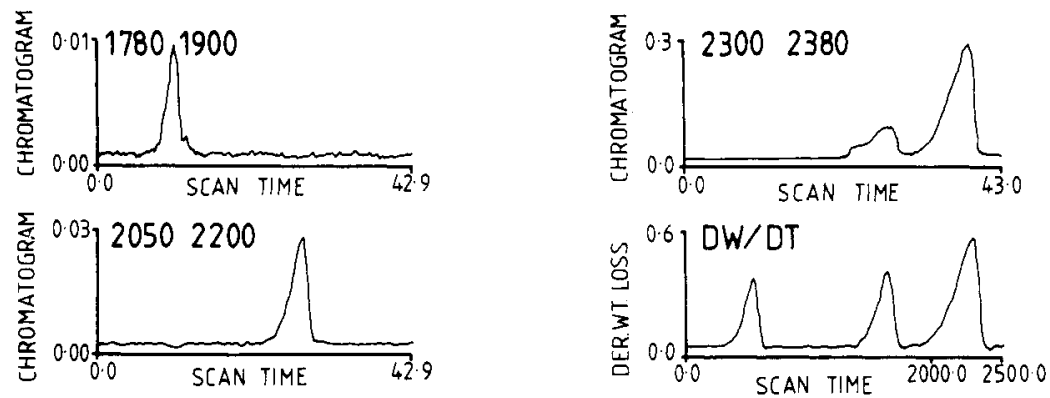

FIG. 2 FTIR AND TG OF CALCIUM OXALATE

\section{POLYMER DEGRADATION}

In the study of the degradation of polymers the combination of TA and FTIR is particularly powerful.

Polytetra fluoro ethylene (PTFE) decomposes in nitrogen in a single reaction to give a material with a spectrum looking like that of tetrafluorethylene. The subtraction of a known spectrum of TFE vapour from the experimental FTIR gave no residue therefore confirming that TFE was the sole product of the thermal degradation of PTFE. If PTFE is heated in air thermo oxidative degradation takes place and in addition to TFE absorption bands corresponding to $\mathrm{CO}_{2}, \mathrm{CO}$, acid fluorides and other oxidation products are observed. 


\section{Fillers}

The decomposition of polymers is often complicated by the presence of fillers. DTA can be used to identify fillers [8].

FTIR however can give a great deal more information as was shown [7] in TGA/FTIR studies on wood which is an important filler for thermoset plastics. The TG trace showed a small loss at $70^{\circ} \mathrm{C}$ and a larger loss around $430^{\circ} \mathrm{C}$. The FTIR shows however that the simple TG curves covers a complex of reactions as the spectra change over the temperature range. Bands due to carbon monoxide, carbon dioxide, water, carbonyl groups and aliphatic and aromatic hydrocarbons are found.

\section{TA AND MASS SPECTROSCOPY}

It has long been realized that the analysis of the gases evolved from TG results would greatly facilitate an understanding of the reaction involved.

Fig 3 shows a combined DTA-TG-MS study of a cured phenolic resin. Phenolic (PF) resins are made by the reaction of phenol and formaldehyde to give compounds that on heating produce a hard, "cured", material. At one time it was thought that PF resins were extensively crosslinked through a series of cyclic compounds but it is now known that much of the rigidity of the cured moulding comes from entanglement of the molecular chains [9]. On the other hand the crosslinking process gives to $\mathrm{PF}$ (and other condensation thermosetting resins) the characteristic mechanical strength that made it pre-eminent amongst insulating engineering materials for many years. PF resins also have excellent fire resistance and they also find application in space vehicles since the thermal degradation of PF resins produces an insulating char which acts as an excellent heat shield.

The TG trace in Fig 3 show little below $400^{\circ} \mathrm{C}$. The lack of sensitivity of TG relative to other TA techniques was pointed out many years ago [10] and can be seen here by comparison with the DTA trace. Comparison of the TG trace with the trace produced for the evolution of Phenol by the mass spectrometer shows that the TG curve is mainly influenced by the loss of phenol which is the largest molecule (m/ z 94) involved.

On the other hand the loss of water $(\mathrm{m} / \mathrm{z} 18)$ and ammonia $(\mathrm{m} / \mathrm{z} 17)$ which do not appear on the TG trace are much more significant because these compounds, and the formaldelyde (m/z 29), are formed by the breaking of crosslinks which have a disproportionate effect on the mechanical properties of PF moulded compounds. Without cross links PF resins are merely very viscous solids, after cross linking they become very strong engineering materials (a tensile strength of $10,000 \mathrm{psi}\left[68.95 \mathrm{MN} / \mathrm{m}^{2}\right]$ is obtainable with appropriate fillers).

The MS traces show that if the PF resin is held at $200^{\circ} \mathrm{C}$ then a catastrophic deterioration in mechanical properties can be expected. The DTA trace also signals a change around $200^{\circ} \mathrm{C}$, DTA is thus a useful indicator of temperatures at which engineering properties may change but MS shows clearly why these changes occur.

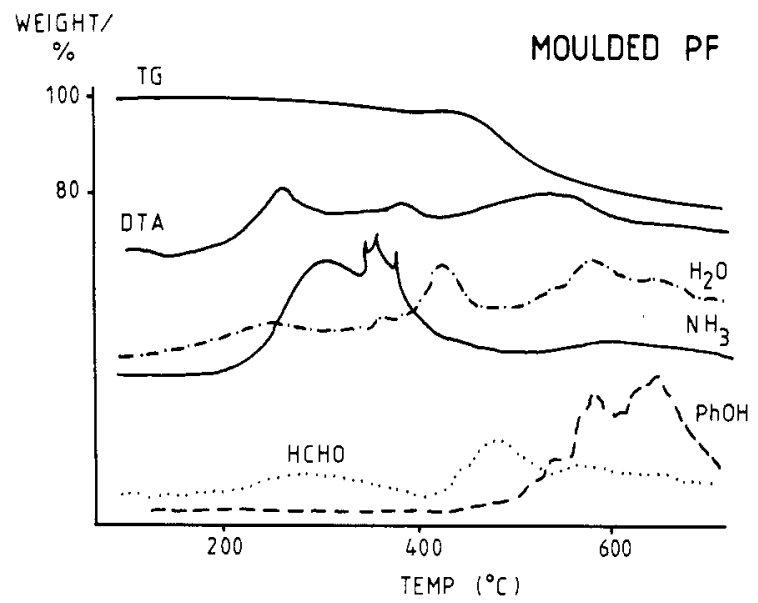




\section{THERMOMECHANICAL TECHNIQUES}

In Thermodilatometry changes in volume or length are measured at varying temperatures under a negligible load. The measurements are often taken by optical or other non-interfering techniques.

The simplest method is to clamp a rod at one end and measure the changes in length at the free end. Thermodilatometry has been used for many years in the study of ceramics but is now being used for polymers. Dilatometry has been a classical technique for the study of the kinetics of polymerisation for many years; thermodilatometry extends this to studies of $T_{\mathrm{g}}$ and crystallisation.

In TMA a sample may be deformed in compression, tension, bending or twisting. The techniques are often much more sensitive than DSC to transitions such as $T_{g}$ which are weakly energetic but show considerable change in mechanical properties.

TMA measurements can be regarded as miniature tensile (compression or flexural) test methods and we can expect to see the influence of TMA on commercial testing apparatus in the near future.

In Dynamic Mechanical Thermal Analysis (DMTA or DMA), also known as dynamic thermomechanometry, an oscillatory stress is imposed on the specimen and the resultant strain is recorded. The stress imposed may be a fixed (variable) frequency or the natural (resonance) frequency of the material.

For a viscoelastic material the dynamic response to an oscillatory stress is governed by a viscous component, which dissipates energy by the relative motion of molecules, and an elastic component, which stores energy until the stress is removed. The strain resulting from the applied stress is also oscillatory with the same frequency but out of phase by an amount dependent on the relative elastic and viscous responses. The elastic and viscous components are related by the expression

$$
E=E^{\prime}+i E^{\prime \prime}
$$

Where $E$ is the appropriate bulk modulus (tensile, shear, flexural, etc.), $E^{\prime}$ is the elastic component or storage modulus, $E^{\prime \prime}$ is the viscous component or complex modulus being the imaginary part of the modulus. For the same reason as for $E^{\prime}, E^{\prime \prime}$ is also known as the 'loss modulus'. The phase difference $\delta$ is known as the 'loss angle'. The bulk modulus is related to the individual components by the expressions

$$
E^{\prime}=E \cos \delta \text { and } E^{\prime \prime}=E \sin \delta
$$

hence:

$$
\tan \delta=E^{\prime \prime} / E^{\prime}
$$

where $\tan \delta$ is known as the loss tangent.

Various types of DMA instruments are available. As for TMA the oscillating stress can be applied in shear, flexure, tension or compression. The sample can either be driven at a constant load or amplitude in which case energy is continuously supplied to the sample and the stress to strain ratio or power load used as the measured variable, or a free vibration can be used, where the sample is initially displaced and the decay in amplitude measured. The frequency of the vibration can either be the natural frequency of the sample (resonance), which will vary with temperature, or an operator specified constant frequency, in which case the oscillation is said to be forced. Finally either the temperature of the test can be held at a constant value while the temperature is changed at a constant rate. Temperature scanning instruments which are driven at the natural frequency of the sample present difficulties in the interpretation of the experimental results as both temperature and frequency change in the course of an experimental run.

The power of DMTA is illustrated [11] by on results for amorphous polystyrene. This shows four transitions $\alpha, \beta, \gamma$ and $\delta$. The higher temperature transition is normally referred to as $\mathrm{T}_{\mathrm{g}}$ or glass-rubber transition which occurs when the main polymer chains are free to rotate and is associated with a substantial fall in mechanical strength. 
As temperatures rise more energy is available and further intra-molecular movements become possible. The temperatures at which these transitions occur are marked by a decrease in modulus and a peak in $\tan \delta$.

In addition to $T_{g}$, DMA shows $\beta, \gamma$ and $\delta$ transitions in PS. The $\beta$ transition is related to movement of the large phenyl rings in the side chain. In the ideal structure for PS it is difficult to identify convincing movements for $\gamma$ and $\delta$. No other technique (apart from DETA) is sufficiently sensitive to detect such transitions. In addition to fundamental studies on transitions DMTA is widely used to quantify the viscoelastic behaviour of polymers which must be taken into account in the production and use of engineering materials.

Because polymers are viscoelastic the properties are dependent on time and temperature. The elastic modulus of a polymer under constant load will decrease with time because the molecular structure rearranges in order to minimize local stresses. In the same way a polymer deformed at a high frequency will exhibit a relatively higher modulus than one deformed at a low frequency.

It is possible to prognosticate the properties of viscoelastic materials to meet the requirements of engineering designers because of the so called super position principle. This states that measurements made at a higher temperature (ie and over a shorter time) may be transposed to a lower temperature and a longer time. it is therefore possible to obtain results applicable to a service life of say 10 years in an experiment lasting a few months. As with all extrapolations however caution is needed when they are used.

DMTA is a convenient method for the study of stress relaxation and thus the expected service life. Oxidation is a major factor in the aging of elastomers and the log of modulus against temperature is shown in Fig 4A and $B$ for nitrile rubber.

Above $30^{\circ} \mathrm{C}$ the rubber became too soft to test. The requirements of the analytical system of many DMTA devices and the mechanical behaviour of the specimens restrict the range of modulus values that can be tested.

From Fig 4 it can be seen from the similarity of the curves for samples aged up to 86 hours that the stress relaxation of the rubbers is dominated by physical factors. After aging for 75 hours the rate of fall in modulus is beginning to decrease; after 86 hours of aging the increase in $\mathrm{T}_{\mathrm{g}}$ means that a significant value for the modulus remains at the end of the experiment. It is clear that the antioxidant is hindering the chemical reaction and so the differences amongst the samples remains small.
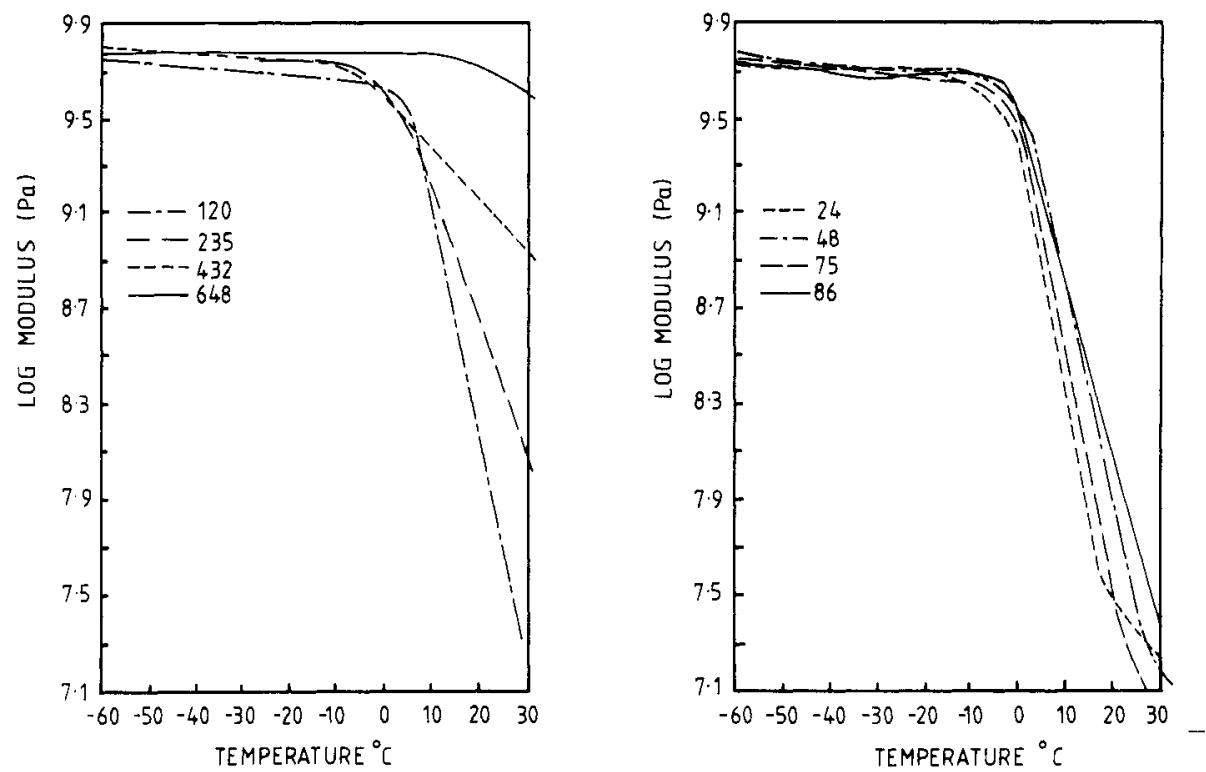

FIG. 4 DMTA OF NITRILE RUBBER 
After 86 hours the effects of chemical relaxation become more obvious, both on the stress relaxation curve and in the change in modulus. At 120 hours a slight stiffening has become apparent while at 235 hours the change in physical properties is obvious. Above this exposure time, the hardening proceeds rapidly, until at 648 hours the material is very heavily degraded, although it has not yet failed the stress relaxation test.

This would indicate that whilst $25 \%$ retention of initial stress may be a convenient criterion for failure in laboratory testing a high value may be preferred when prognosticating the service life of an elastomer [12].

\section{THERMAL DEGRADATION OF PVC}

Polyvinyl Chloride (PVC) [poly-1-chloroethylene] is one of the most important plastics now available, being second only to polythene. Interestingly it is one of the least thermally stable commercial polymers since it begins to degrade by means of an unzipping reaction above $70^{\circ} \mathrm{C}$. Whether such a material if newly discovered today would ever emerge from the research laboratory is doubtful to say the least.

Thermal analysis techniques were shown [13] to have no significant advantages over standard methods for the degradation of PVC so a more fundamental change was made.

The onset of degradation is of major interest. To study this onset requires high sensitivity and close control over reaction conditions. It was decided to replace the standard DTA and TG furnaces with a fluidized bed [14]. This has the following advantages:

i powdered samples avoid the effects of the solvents or special forms (films, discs, or rods) that vary the rates of diffusion.

ii the particles do not sinter above the $\mathrm{T}_{\mathrm{g}}$ and thus variations in rates of diffusion are obviated [14].

iii precise control of the temperature in the reaction zone is easily achieved and concentration and temperature gradients are eliminated.

iv the reactor is made of glass to avoid reactions with metals and to allow the observation of colour changes in the sample.

v the hydrogen chloride released is quickly absorbed in ultra-pure water.

Initially the effects of diffusion in the atmosphere surrounding the particles were examined. Once these had been established experimental conditions were chosen to eliminate the effects of this external diffusion.

Intra particle diffusion was then studied with reference to the size and porosity of the specimens. Finally the effect of varying the temperature between 80 and $200^{\circ} \mathrm{C}$ on the course of the thermal degradation was investigated. This last effect was also studied in a thermobalance.

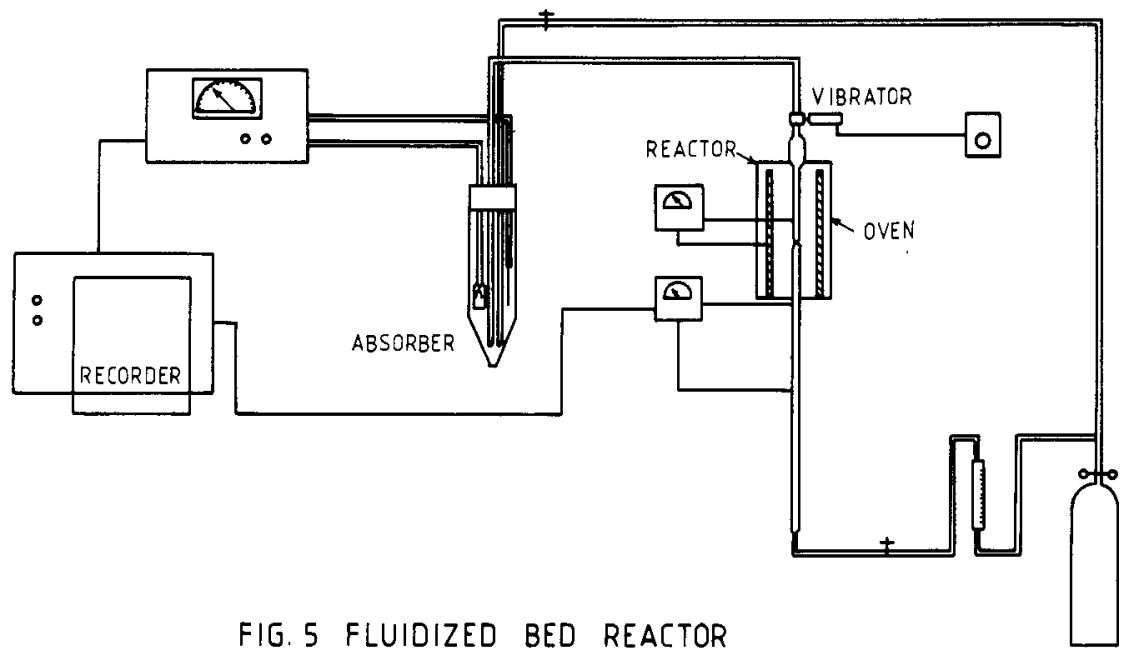


To make absolutely sure that sintering did not occur above the glass transition temperature $\left(\mathrm{T}_{\mathrm{g}}\right)$ of the PVC an inert powder was added to separate the PVC particles.

Pure nitrogen ( $99.9992 \%$ ), with less than $1 \mathrm{ppm}$, of oxygen was used as the fluidizing gas.

The graphs of conductivity versus time show a small but abrupt departure from the asymptotic value of the degassed ultra-pure water when the temperature is raised over $80^{\circ} \mathrm{C}$, and a milder but progressive slope ending in a almost straight line when the first hydrogen chloride released is detected. This starting zone depends on the final temperature reached, but always ends before reaching a conductivity of 0.2 S. Taking this last value as the first clear indicator of degradation the sensitivity of the method for a normal run, involving $0.600 \mathrm{~g}$ of PVC and 0.4001 of absorbing water, would be $13 \mathrm{ppm}$. This sensitivity lies among the highest reported and, if necessary, could be easily increased using bigger samples or reducing the absorbing water. Hence good correspondence between the amount of hydrogen chloride detected at this early stage and that given off by the sample can be assumed. The conductimetric detection occurs before any appreciable change in colour can be noticed in the sample.

The Fluidized technique enables one to study the PVC thermal degradation kinetics at low temperatures $\left(80^{\circ} \mathrm{C}\right)$, and with a 30 fold increase in sensitivity. Accurate detection of the hydrogen chloride evolved is possible long before the sample changes its colour. Much of the disagreement amongst workers who have used powdered samples in crucibles or in fixed beds can be attributed to neglecting diffusion, especially of intra particular diffusion. The Arrhenius plot yields an apparent activation energy of 29 $\mathrm{Kcal} / \mathrm{mol}$.

The use of a fluidized bed in conjunction with MS or FTIR should greatly increase the reliability of kinetic results in TA.

\section{CONCLUSIONS}

Thermal analysis of Polymers has advanced rapidly in the last 25 years. In conjunction with other techniques it will be of increasing value to polymer scientists and will be an essential part of any laboratory for the study of plastics and indeed other materials.

\section{REFERENCES}

[1] Manley. T.R. "DTA and its application to Polymer Science" in Techniques of Polymer Science Monograph, 17, Soc. Chem. Ind., London (1963).

[2] Manley, T.R., "Thermal Stability of hexamethylol melamine", Polymer J. Japan 4, 111 (1972).

[3] Manley, T.R. and Scurr, G. "DTA of U.V. cured resins", J.O.C.C.A. 66, 43-48 (1983)

[4] B.R. Glennie and T.R. Manley, "Measurement of smoke from smouldering polymers", Soc. Plastics Engineers 34th ANTEC Atlantic City, p. 396 (1976).

[5] Fellgett, P., J. Phys. Radn. 19187 (1958)

[6] Manley T.R. and Williams D.A, I.U.P.A.C. Macromolecular Conference Prague, A529 (1965).

[7] D.A.C. Compton, "On line F.T.I.R. analysis of Gaseous Effluent from a Thermogravimetric Analyser", in press.

[8] Manley, T.R., "Characterization of filled amino resins by thermal analysis", Trans. Plastics Inst. p. 525 (1967).

[9] N.J. Megson, Phenolic Resin Chemistry, Butterworth London (1958).

[10] T.R. Manley in "H.V applications of Epoxy Resins", Ed. M. Billings, U.M.I.S.T. press (1967).

[11] R.E. Wetton, "Dynamic Mechanical Measurements and their application to Polymer Systems", Analyt. Proceedings, R.S.C. London October 1981 p. 417.

[12] Manley, T.R. and Clark L.J., "Stress Relaxation Studies", Proc. Int. Rubber Con. Goteborg 1, p. 103. (1986).

[13] Guy. A., M.Sc. Thesis Newcastle Polytechnic (1980).

[14] J.A. Gracia Martinez, F. Gutierrez Fernandez, T.R. Manley, "Initial thermal stability of PVC", Brit. Polymer J., 18, 201 (1986). 